\title{
Effects of Heating on Electrical and Spectral Properties of In/CdTe/Au X- and $\gamma$-ray Detectors with a Schottky Barrier or Laser-induced $p-n$ Junction
}

\author{
Junichi Nishizawa, ${ }^{1 *}$ Volodymyr Gnatyuk, ${ }^{2}$ Kateryna Zelenska, ${ }^{3}$ and Toru Aoki ${ }^{1,3,4}$ \\ ${ }^{1}$ Graduate School of Science and Technology, Shizuoka University, \\ 3-5-1 Johoku, Naka-ku, Hamamatsu 432-8011, Japan \\ ${ }^{2}$ V.E. Lashkaryov Institute of Semiconductor Physics of the National Academy of Sciences of Ukraine, \\ Prospekt Nauky 41, Kyiv 03028, Ukraine \\ ${ }^{3}$ Research Institute of Electronics, Shizuoka University, 3-5-1 Johoku, Naka-ku, Hamamatsu 432-8011, Japan \\ ${ }^{4}$ ANSeeN Inc. 3-5-1 Johoku, Naka-ku, Hamamatsu 432-8011, Japan
}

(Received August 7, 2020; accepted November 2, 2020)

Keywords: $\mathrm{CdTe}, \mathrm{X} / \gamma$-ray detector, $p-n$ junction, $I-V$ characteristics, laser-induced backside doping

The CdTe semiconductor has been used for X- and $\gamma$-ray imaging detectors owing to its sensitivity to ionizing radiation, high energy and spatial resolution, and ability to operate at room temperature. However, it is challenging to grow large-diameter CdTe. Therefore, we aim to develop large-area high-resolution X-ray imaging sensors by tiling small CdTe wafers. Postprocessing, bonding, and tiling require the heating of detectors; therefore, it is important to develop high-thermal-tolerance radiation sensors. We have fabricated two types of In/CdTe/Au diode detector (with a Schottky barrier and a $p-n$ junction) using identical crystals and the same technological procedures except laser irradiation, and examined their resistance to heat treatment. The diodes with an $\mathrm{In} / \mathrm{CdTe}$ rectifying contact and a $p-n$ junction, formed by the laser-induced backside doping of the CdTe subsurface layer with In, had similar electrical and spectral characteristics, which did not significantly change after heating at temperatures below the In melting point $\left(156^{\circ} \mathrm{C}\right)$. The Schottky diodes, subjected to heating at 200 and $300{ }^{\circ} \mathrm{C}$, exhibited the deterioration of the electrical characteristics (reverse dark current increased) and lost their detection ability. In contrast, the $p-n$ junction diodes demonstrated highly stable $I-V$ characteristics, and the measured ${ }^{241} \mathrm{Am}$ radioisotope emission spectra did not degrade even after melting and solidifying the In electrode.

\section{Introduction}

Owing to the direct conversion of photons to electrical charges (photoeffect), semiconductor detectors have great advantages, in comparison with other types of ionization radiation sensor such as gas-based detectors or scintillators, and their main features are high energy resolution, imaging capabilities, and the ability to fabricate compact detecting devices. ${ }^{(1)}$ Among the semiconductors used for $\mathrm{X}$ - and $\gamma$-ray detectors, CdTe is the most attractive and promising

*Corresponding author: e-mail: nishizawa.junichi.15@shizuoka.ac.jp https://doi.org/10.18494/SAM.2020.3054 
material because of its suitable properties. ${ }^{(2,3)}$ The high atomic numbers of components $\left(Z_{\mathrm{Cd}}=48, Z_{\mathrm{Te}}=52\right)$ and the large density of the compound $\left(5.8 \mathrm{~g} / \mathrm{cm}^{3}\right)$ provide excellent photon absorption efficiency and stopping power, and therefore, a larger attenuation coefficient than $\mathrm{Si}, \mathrm{Ge}$, and other semiconductors used for high-energy radiation detectors. ${ }^{(2,3)} \mathrm{Si}$ detectors cannot detect X-rays above $100 \mathrm{keV}$ because of low quantum efficiency, and for Ge detectors, liquid nitrogen or Peltier cooling is required because of thermally induced leakage current. In CdTe, the wide band gap $\left(E_{\mathrm{g}}=1.46 \mathrm{eV}\right)$ and, hence, the high resistivity $\left(\sim 10^{9} \Omega \cdot \mathrm{cm}\right)$ along with appropriate charge transport characteristics allow CdTe-based detectors to operate without cryogenic cooling and make it possible to achieve high detection efficiency, high energy resolution, and fast timing response. ${ }^{(2,3)}$

Thus, owing to an optimal set of the physical characteristics of CdTe semiconductors, $\mathrm{CdTe}$ is the basic material for high-energy radiation direct-conversion detectors operating at room temperature and covering a wide energy range from $60 \mathrm{keV}$ to $6 \mathrm{MeV}$. CdTe-based detectors exhibit high sensitivity to ionizing radiation and offer satisfactory spatial and energy resolutions. They are compact and, therefore, applicable to the fabrication of various portable Xand $\gamma$-ray radiation detection and imaging instruments. Such devices are necessary for various fields of industry, science, medicine, security, ecology, astronomy, and so forth, requiring the identification and localization of nuclear radiation sources (e.g., spectrometry, environmental monitoring, and control of nuclear objects) or visualization and imaging (e.g., nondestructive inspection, material identification, medical examination, customs control, baggage checking, and space object imaging). ${ }^{(2,3)}$ Researchers in fields related to these applications have strived to achieve high detection sensitivity and spectral energy resolution to enable short-duration data collection and high-contrast imaging at low exposure rates. ${ }^{(2-7)}$

Generally, CdTe-based detectors are fabricated as diodes with a high potential barrier to reduce dark current because ohmic CdTe detectors suffer from enhanced dark current at higher biases and cannot efficiently operate at applied voltages above $100 \mathrm{~V} .^{(2,3)}$ Diode-type detectors, fabricated as structures with a Schottky barrier or a built-in $p-n$ junction, which operate at larger reverse bias voltages, exhibit higher charge collection efficiency and energy resolution than ohmic contact. ${ }^{(4-7)}$ Nevertheless, CdTe has several disadvantages, including the small size of its wafers. Currently available growth technologies cannot produce large CdTe crystals without numerous structural defects. For example, C9732DK-11 flat-panel CMOS scintillator image sensors with dimensions of $12 \times 12 \mathrm{~cm}^{2}$, developed by Hamamatsu Photonics, are currently used for precision dental examinations; ${ }^{(8)}$ that is, $12 \times 12 \mathrm{~cm}^{2}$ is the recommended size of sensors in the industry. However, CdTe single crystals with a maximum diameter of only $10 \mathrm{~cm}$ can be grown by Acrorad Co., Ltd., making their applicability in sensors difficult. ${ }^{(9)}$

Assembling large sensor systems by tiling pixel detectors and 3D chip stacking requires solder bumping and wire bonding fabrication. During these processes, the detectors are subjected to heating to temperatures of up to $200{ }^{\circ} \mathrm{C} .{ }^{(10-14)}$ CdTe Schottky diode detectors, which are widely used for $\mathrm{X}$ - and $\gamma$-ray imaging, are extremely sensitive to postprocessing operations (e.g., mechanical and thermal bonding, and soldering), which limit the technological procedures available in large-sensor module fabrication. It is known that a Schottky barrier is formed directly at the metal-semiconductor interface, and its properties are determined by the 
surface states of the semiconductor crystal and the quality of the deposition of a defined metal (such as In in this study). ${ }^{(15)}$ Thermal, mechanical, ultrasound or other types of treatment, usually used during bonding procedures, can easily destroy the specific state, which was created to form a high Schottky barrier and, therefore, to deteriorate the electrical and spectral characteristics of Schottky diode-type detectors. Therefore, diodes with different types of barrier that are more resistant to external influences, particularly to heating, and have stable characteristics are desirable for practical applications. It is considered that diodes with a builtin electrical junction, formed inside the semiconductor at a certain depth, are promising. ${ }^{(5-7)}$

Therefore, in this study, we examined the thermal stability of the electrical and spectral properties of In/CdTe/Au X-and $\gamma$-ray detectors fabricated on the basis of semi-insulating $p$-like CdTe semiconductor crystals using two approaches: (i) the conventional method of forming a Schottky barrier at the In/CdTe interface by the vacuum evaporation of an In electrode and (ii) the creation of a $p-n$ junction near the In/CdTe interface by the laser-induced backside doping of a CdTe layer with In atoms.

\section{Materials and Methods}

Diode-type structures with a Schottky barrier and a $p-n$ junction were fabricated using (111)-oriented $p$-like CdTe semiconductor single-crystal wafers with dimensions of $5 \times 5 \times 0.75 \mathrm{~mm}^{3}$. The wafers had been chemically cleaned and etched using a standard procedure: they were cleaned in acetone and methanol and then subjected to polishing etching in a $5 \% \mathrm{Br}-$ methanol solution. ${ }^{(7)}$ Two types of electrode were used: (1) an In electrode in the case of the Schottky barrier or $p-n$ junction on the Te-terminated surface ( $B$-face) and (2) a $\mathrm{Au}$ electrode with a near-ohmic contact on the Cd-terminated surface ( $A$-face). The In and $\mathrm{Au}$ electrodes were deposited in a vacuum chamber at a pressure of $3.4 \times 10^{-4} \mathrm{~Pa}$. The thicknesses of the In and Au films were $200 \mathrm{~nm}$. Thus, In/CdTe/Au barrier structures with a rectifying In/ CdTe contact were obtained and then examined as Schottky diode X-and $\gamma$-ray detectors.

The In/CdTe/Au $p-n$ junction diodes were fabricated by the laser irradiation of the $\mathrm{In} / \mathrm{CdTe}$ structures before $\mathrm{Au}$ electrode deposition. A $p-n$ junction was created in the CdTe subsurface layer by laser-induced backside doping with In atoms from the deposited In electrode, which served as the dopant source. ${ }^{(16-19)}$ A schematic of the experimental setup for the laser-induced doping procedure is shown in Fig. 1. A Nd:YAG laser with a pulse duration of $\tau=20$ ns was employed. The laser operated in the repetition mode with a repetition rate of $10 \mathrm{~Hz}$. Laserinduced backside doping was conducted after the In film deposition.

The In/CdTe interface was irradiated through the CdTe crystal using laser pulses at a wavelength $(\lambda)$ of $1064 \mathrm{~nm}$. Infrared laser radiation was attenuated by less than $1 \%$ in CdTe and strongly absorbed by the In film, resulting in the local heating or melting of a thin In layer at the In/CdTe interface. ${ }^{(16)}$ A simultaneously generated second harmonic of laser radiation with a wavelength of $532 \mathrm{~nm}$ was cut using a selective mirror and a color filter (Fig. 1). An expander provided a laser spot with a diameter of $12 \mathrm{~mm}$; thus, radiation covered the entire surface of the sample with a size of $5 \times 5 \mathrm{~mm}^{2}$. A homogenizer was used to flatten the laser spatial intensity profile. Neutral density filters were used to control the laser pulse intensity. 


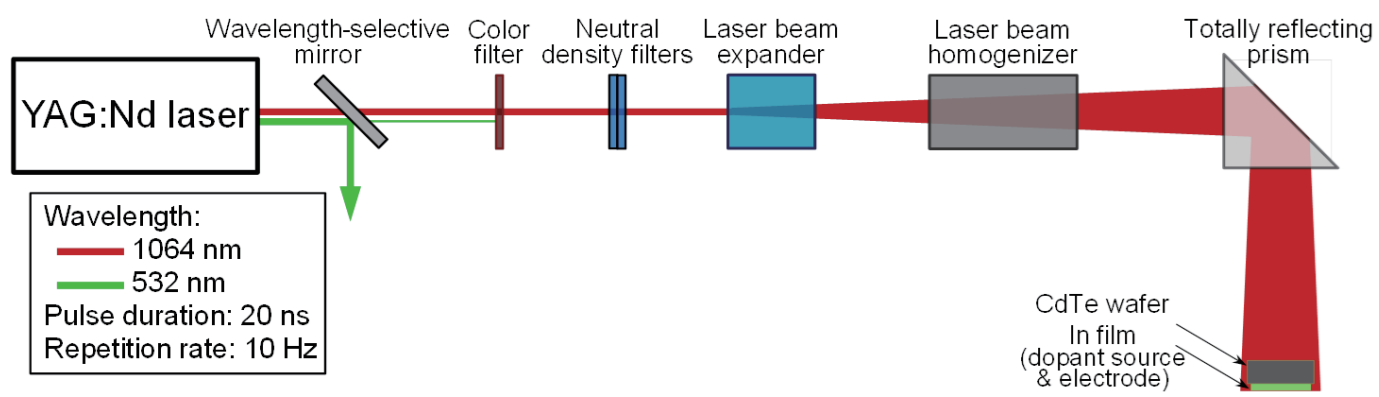

Fig. 1. (Color online) Schematic diagram of optical setup for backside laser irradiation of In/CdTe interface.

The electrical properties of the obtained diodes with the $p-n$ junction depended on the laser energy and the number of laser pulses. The optimal conditions for laser-induced backside doping were studied and estimated in our earlier publications. ${ }^{(16-19)}$ The selected laser energy in the experiments was $E=11 \mathrm{~mJ}$ for which the temperature of the In surface at the $\mathrm{In} / \mathrm{CdTe}$ interface increased up to near the In melting point. The number of laser pulses, used for backside doping, was 30 .

The heat treatment of the obtained Schottky and $p-n$ junction diodes was conducted in vacuum at temperatures of 100,200 , and $300{ }^{\circ} \mathrm{C}$ for $1 \mathrm{~h}$. The samples were cooled to room temperature, following which electrical and spectral measurements were obtained. The applied bias voltage in the $I-V$ characteristic measurements varied over a wide range. As known, diode-type $\mathrm{X}$ - and $\gamma$-ray detectors operate in the reverse bias mode. ${ }^{(2,3)}$ Therefore, the voltage dependences of the dark current of the fabricated $\mathrm{In} / \mathrm{CdTe} / \mathrm{Au}$ diodes with a Schottky barrier or a $p-n$ junction were studied under reverse bias in the range from -500 to $0 \mathrm{~V}$. The reverse current in the In/CdTe/Au diodes flowed when the In contact was biased positively with respect to the $\mathrm{Au}$ one (ohmic contact).

For spectral measurements, an ANS-CSA1A100-02-NJ preamplifier and an ANSHSDMCA4M4N17-NJ multichannel analyzer (ANSeeN, Inc.) were used. The In and Au electrodes were connected to the signal line and shield, respectively. An ${ }^{241} \mathrm{Am}$ radioisotope was employed as the high-energy radiation source. The energy of the ${ }^{241} \mathrm{Am}$ spectrum peak is $59.5 \mathrm{keV} .^{(2,3)}$ Such radiation was significantly absorbed by the CdTe semiconductor. This allowed us to examine the fabricated In/CdTe/Au detectors even if they did not have a high sensitivity. Certainly, in the isotope emission spectra measurements, the relative positions of the detector and X-ray emission source play a significant role. ${ }^{(1-3)}$ In our study, the ${ }^{241} \mathrm{Am}$ radioisotope was placed over the In electrode for all the In/CdTe/Au detectors, and the distance between the radiation source and the samples was the same during the spectral measurements.

The $\mathrm{In} / \mathrm{CdTe} / \mathrm{Au}$ diode detectors were reverse-biased at $V=-800 \mathrm{~V}$. The rather high bias voltage was applied to extend the depletion region (space-charge region width), overcome the charge losses and, thus, provide the full collection of photogenerated charge carriers. ${ }^{(20)}$ Generally, X- and $\gamma$-ray spectral measurements for diode-type detectors are carried out at high electrical field strength to obtain a higher energy resolution and an appropriate peak channel 
position in the radioisotope spectra. ${ }^{(9,12,20,21)}$ Furthermore, CdTe diode detectors suffer much less from the "polarization" phenomenon if a higher bias voltage is applied. ${ }^{(21)}$

The fabrication procedures of $\mathrm{In} / \mathrm{CdTe} / \mathrm{Au}$ diodes and the measurements of the electrical and spectral properties of the diode-type X- and $\gamma$-ray detectors were carried out at room temperature.

\section{Results and Discussion}

Thus, two types of In/CdTe/Au diode structure were developed using identical CdTe crystals, the same surface processing, the same metals (In and $\mathrm{Au}$ ) for electrical contact, and the same electrode deposition technique (vacuum evaporation), i.e., the same technological fabrication procedures were employed except laser irradiation. Unirradiated $\mathrm{In} / \mathrm{CdTe} / \mathrm{Au}$ structures were typical Schottky diodes. Metal-CdTe Schottky diodes have been generally fabricated and used as X- and $\gamma$-ray detectors. ${ }^{(2-5,9,12,20,21)}$ On the other hand, In/CdTe/Au barrier structures formed by frontside ${ }^{(5-7)}$ or backside laser irradiation ${ }^{(16-19)}$ have been considered as $p-n$ junction diodes, which have also shown high detection efficiency.

Figure 2 shows the schematic of the structures of $\mathrm{In} / \mathrm{CdTe} / \mathrm{Au} \mathrm{X}$ - and $\gamma$-ray detectors formed as (a) a Schottky diode (without laser irradiation) and (b) a $p-n$-junction diode by backside laser irradiation. $\mathrm{X}$ - and $\gamma$-photons, absorbed by the semiconductor as a result of the photoelectric effect, generated electron-hole pairs in the CdTe crystal. Nonequilibrium electrons and holes were swept towards the appropriate electrodes by the applied electric field. The In film was the collecting electrode formed with (i) a Schottky contact in the diodes fabricated without laser processing and (ii) an ohmic contact to the thin low-resistivity $n$-CdTe layer, doped with In atoms (donors), in the $p-n$ junction diodes created by backside laser irradiation [Fig. 2(a) and 2(b), respectively]. The drift of holes towards the cathode (Au electrode) and electrons towards the anode (In electrode) induced a charge signal at the collecting electrode, which was measured with appropriated front-end electronics.

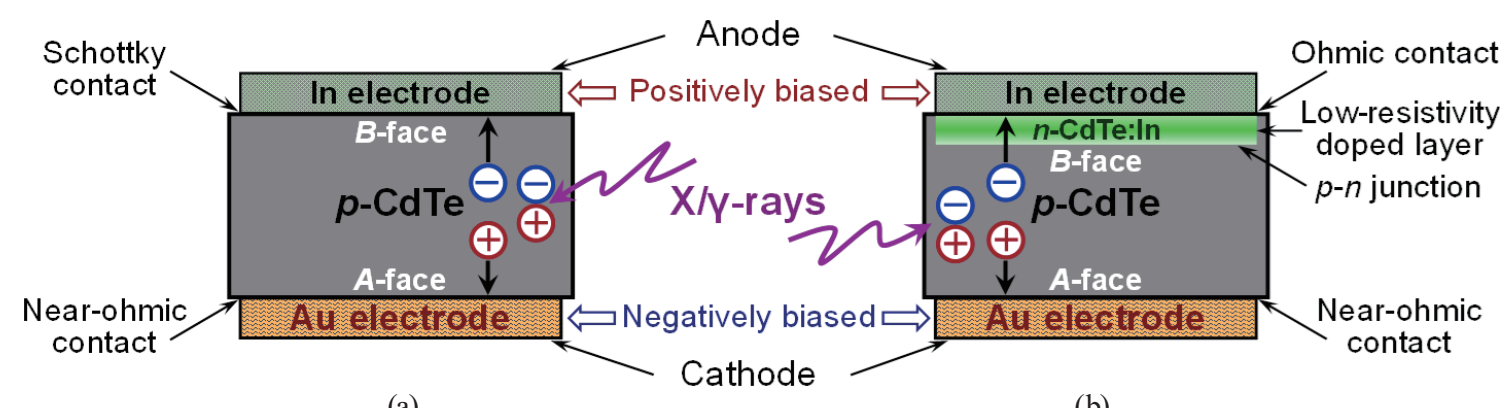

(a)

(b)

Fig. 2. (Color online) Illustration of structures of two In/CdTe/Au X-and $\gamma$-ray sensors formed (a) without laser irradiation (Schottky diode) and (b) with laser-induced backside doping ( $p-n$ junction diode). The generation of electron-hole pairs and charge collection in CdTe crystals in the reverse biased diodes under interaction with X-and $\gamma$-ray photons (photoeffect) are also schematically shown. 
In this study, for the laser-induced backside doping of a thin CdTe layer and the creation of a $p-n$ junction near the $\mathrm{In} / \mathrm{CdTe}$ interface, we used the laser energy that caused the heating of the In surface, adjoining to the CdTe layer, to near the In melting point. The temperature of the In surface was calculated in the vicinity of the In/CdTe interface under irradiation with a nanosecond laser pulse ( $\lambda=1064 \mathrm{~nm}, \tau=20 \mathrm{~ns})$ according to the simulation model based on the thermal conduction equation. ${ }^{(16,22)}$ The calculated time dependences of the In film temperatures at the $\mathrm{In} / \mathrm{CdTe}$ interface under irradiation with a laser pulse duration of $20 \mathrm{~ns}$ and energies of 5 and $11 \mathrm{~mJ}$ are depicted in Fig. 3. Laser pulses with $E=11 \mathrm{~mJ}$ were used for the backside doping and fabrication of $\operatorname{In} / \mathrm{CdTe} / \mathrm{Au} p-n$ junction diodes.

The electrical and spectral characteristics of the fabricated In/CdTe/Au diode-type detectors were investigated before and after the heat treatment of the samples. Most of the $p-n$ junction diodes had similar or lower reverse dark currents than the Schottky diodes. To compare the thermal stability features of both types of diode detector, we selected the Schottky and $p-n$ samples with similar initial reverse currents at the same reverse bias voltages. The typical $I-V$ characteristics of the reverse-biased Schottky diode and the diode with a $p-n$ junction before and after heat treatment at 100,200, and $300{ }^{\circ} \mathrm{C}$ for $1 \mathrm{~h}$ are shown in Fig. 4.

As depicted in Fig. 4(a), the reverse current in the Schottky diode after heat treatment conspicuously increased. In particular, a marked increase in reverse current was observed when the In/CdTe/Au Schottky diode was subjected to heating at temperatures higher than the In melting point $\left(T_{\mathrm{In}}=156^{\circ} \mathrm{C}\right)$. As seen in Fig. 4, the current increased in the sample after heating at $T=200{ }^{\circ} \mathrm{C}$ (curve 3) and by more than two orders of magnitude in the sample subjected to heating at $T=300{ }^{\circ} \mathrm{C}$ (curve 4). Electrical noises also markedly increased. These effects resulted in the deterioration of the $\mathrm{In} / \mathrm{CdTe}$ Schottky contact. Consequently, heating and especially melting and the following solidification of the In electrode led to the damage of the Schottky barrier at the In/CdTe interface [Fig. 2(a)]. It is expected and understandable that the

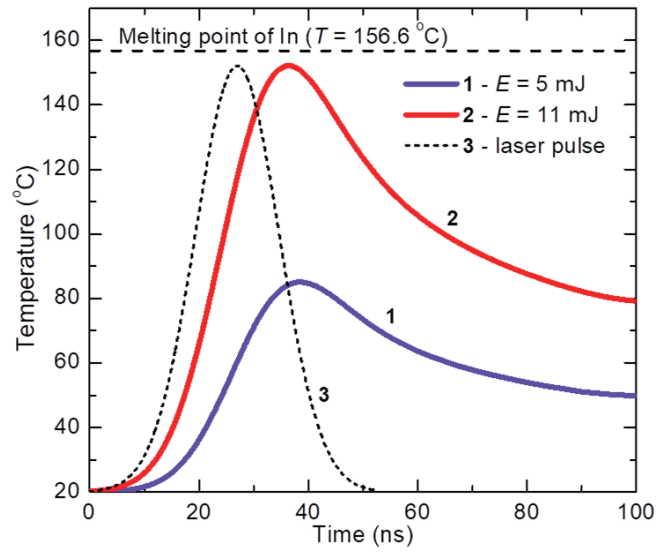

Fig. 3. (Color online) Calculated time dependences of temperature of In film surface at In/CdTe interface under irradiation with laser pulse energies of $5 \mathrm{~mJ}$ (curve 1) and $11 \mathrm{~mJ}$ (curve 2). The In melting point (at $T=156{ }^{\circ} \mathrm{C}$ ) is indicated by the dashed line, and the laser pulse profile is represented by curve 3 . 


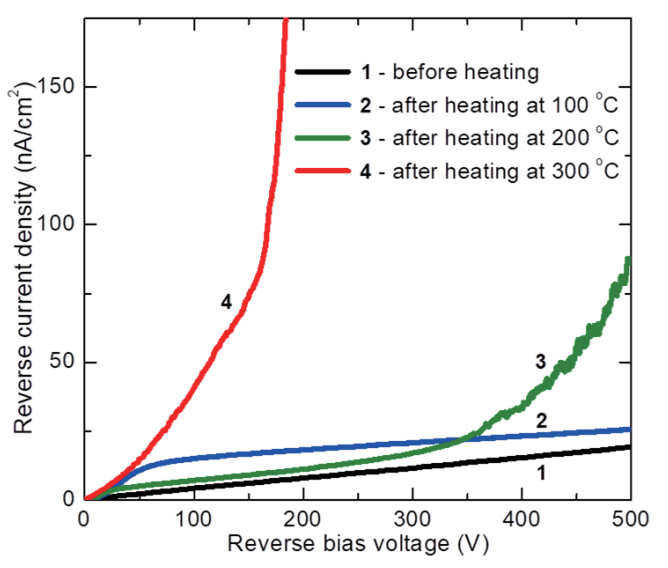

(a)

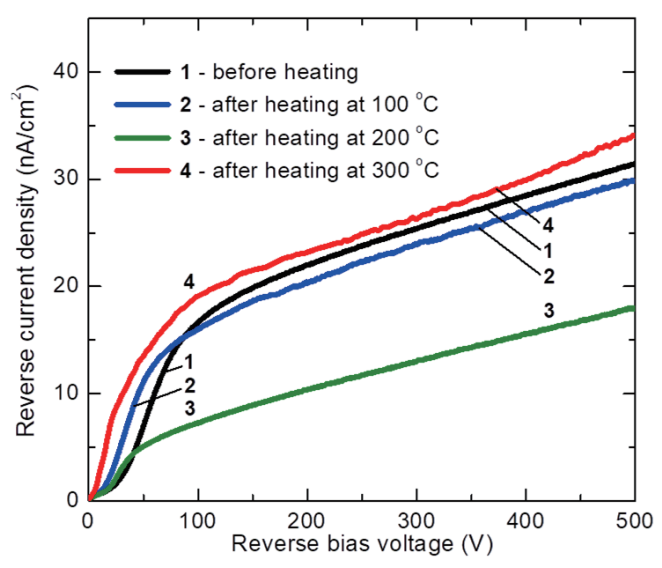

(b)

Fig. 4. (Color online) $I-V$ characteristics of reverse-biased $\mathrm{In} / \mathrm{CdTe} / \mathrm{Au}$ diodes with (a) Schottky barrier and (b) $p-n$ junction before (curve 1 ) and after (curves $2-4$ ) heat treatment of samples at different temperatures.

melted and solidified In contact partly loses its rectifying ability, i.e., reverse current increases [Fig. 4(a), curves 2 and 3].

At the same time, the In/CdTe/Au diode with a $p-n$ junction was sufficiently resistant to heat treatment; i.e., the reverse current did not increase [Fig. 4(b)]. Even after heating the samples at $T>T_{\text {In }}$, i.e., when the In electrode was melted and solidified, the current was lower (curve 3) or only slightly higher (curve 4) than that in the heat-untreated diode (curve 1) [Fig. 4(b)]. It was revealed that heat treatment did not affect the In-doped CdTe region near the In/CdTe interface nor did it deteriorate the built-in $p-n$ junction. The key feature is that the $p-n$ electrical junction with a high potential barrier is formed not at the In/CdTe interface, as in the case of a Schottky barrier in the In/CdTe/Au diodes [Fig. 2(a)], but in the underlying semiconductor region, i.e., inside the crystal at the boundary of the $n$-type CdTe:In layer and the bulk part of $p$-type CdTe [Fig. 2(b)]. The thickness of the In-doped CdTe layer has been estimated as $\sim 50 \mathrm{~nm}$. Heating the $\mathrm{In} / \mathrm{CdTe} / \mathrm{Au}$ diodes up to 200 or $300{ }^{\circ} \mathrm{C}$ cannot destroy a $p-n$ junction located at such depth in the semiconductor because the melting point of CdTe is much higher $\left(T_{\mathrm{CdTe}}=1090{ }^{\circ} \mathrm{C}\right)$.

Furthermore, the decrease in the reverse current of the $p-n$ junction diodes after heat treatment at $T=200{ }^{\circ} \mathrm{C}$ improved the performance of the $\mathrm{In} / \mathrm{CdTe} / \mathrm{Au}$ detectors fabricated by laser-induced backside doping [Fig. 4(b)]. The ideality factors of the obtained Schottky and $p-n$ diodes were estimated from the forward bias parts of the $I-V$ characteristics in the range of $[0.05 \mathrm{~V}$, $0.2 \mathrm{~V}]$, where $V>k T / q$ at $T=297 \mathrm{~K} \cdot{ }^{(15,23-25)}$ The results are presented in Table 1 .

As reported in the literature, the ideality factor is equal to 1 for ideal $p-n$ junctions, whereas for $p$-i- $n$ junctions, it is equal to approximately $2 .^{(24,25)}$ The $\mathrm{In} / \mathrm{CdTe} / \mathrm{Au} p-n$ junction diodes, obtained by the laser-induced backside doping of the subsurface layer of the $p$-like CdTe crystals with an $n$-type dopant (In atoms), showed an ideality factor of approximately 2.8 , which was higher than that for $p-n$ diodes but close to that for $p$-i- $n$ diodes. ${ }^{(25)}$ This may be related to the intrinsic nature of the CdTe semiconductor wafers. ${ }^{(4)}$ The ideality factor of the In/CdTe/Au 
Table 1

Ideality factors of $\mathrm{In} / \mathrm{CdTe} / \mathrm{Au}$ diodes with Schottky barrier and $p-n$ junction.

\begin{tabular}{lcc}
\hline Temperature $\left({ }^{\circ} \mathrm{C}\right)$ & Schottky barrier diode & $p-n$ junction diode \\
\hline Before heat treatment & 3.9 & 2.9 \\
100 & 4.8 & 2.8 \\
200 & 5.9 & 2.8 \\
300 & 33 & 2.7 \\
\hline
\end{tabular}

Schottky diodes was higher than that of the $p-n$ junction diodes, and it increased in the samples after heat treatment, particularly at $T=300{ }^{\circ} \mathrm{C}$ (Table 1). This was due to the significant changes in the recombination processes because of the deterioration of the Schottky barrier. ${ }^{(15,23)}$

The spectroscopic performance of the fabricated In/CdTe/Au diode X-and $\gamma$-ray detectors was tested by measuring the emission spectra of an ${ }^{241} \mathrm{Am}$ radioisotope at room temperature. The Schottky and $p-n$ diodes before heating, whose electrical characteristics did not differ markedly, showed similar (almost identical) spectra taken under the same conditions. A spectrum typical for In/CdTe/Au detectors with either a Schottky barrier or a $p-n$ junction is shown in Fig. 5 (curve 1). The detection efficiencies and energy resolutions of both types of $\mathrm{In} / \mathrm{CdTe} / \mathrm{Au}$ diode detector were almost the same. The energy resolution [full width at half maximum (FWHM)] for the $59.5 \mathrm{keV}$ peak was about 3.2-3.3 $\mathrm{keV}(6.5-6.9 \%)$. The ${ }^{241} \mathrm{Am}$ isotope spectra measured using both types of $\mathrm{In} / \mathrm{CdTe} / \mathrm{Au}$ diode detector after heating at $T<T_{\text {In }}$ were slightly worse or better than those shown in Fig. 5. Slight differences in spectral characteristics were associated with the initial detector performance characteristics (e.g., morphology and structure inhomogeneities in different CdTe wafers, and deviations in surface processing and electrode deposition).

It was revealed that, after heat treatment at $T>T_{\text {In }}$, the In/CdTe/Au Schottky diode detectors significantly degraded and did not record radioisotope spectra. The main reason for the deterioration of the In/CdTe Schottky contact properties is that Schottky barrier is formed just at the crystal surface as a metal-semiconductor interface and can be easily damaged during heating, particularly when the In electrode is melted and solidified, which markedly changes the In/CdTe interface states. The significantly increased leakage current [reverse dark current, Fig. 4(a), curves 3 and 4] and other thermally induced degradation phenomena in the rectifying contact (e.g., lowering the potential barrier height and increasing electrical noise) strongly affect the signal-to-noise ratio of the $\mathrm{In} / \mathrm{CdTe} / \mathrm{Au}$ Schottky-diode detector subjected to heating at 200 and $300{ }^{\circ} \mathrm{C}$.

In contrast to the $\mathrm{In} / \mathrm{CdTe} / \mathrm{Au}$ Schottky diodes, both the untreated and heat-treated (even at $T>T_{\text {In }}$ ) In/CdTe/Au diode detectors with a laser-induced $p-n$ junction almost did not show a change in detection efficiency. The peak in the ${ }^{241} \mathrm{Am}$ isotope spectrum, obtained using the $p-n$ junction diode detector after heating at $T=300^{\circ} \mathrm{C}$, slightly shifted toward the lower-energy side, and the energy resolution (FWHM) was equal to $3.4 \mathrm{keV}$ (curve 2), whereas the FWHM of the untreated sample was 3.2 (curve 1) (Fig. 5). These insignificant changes could be due to Compton scattering effects. ${ }^{(9)}$

The fact that the electrical and spectral characteristics of the In/CdTe/Au $p-n$ junction diode were stable and did not deteriorate after heating the sample at temperatures higher than the In 


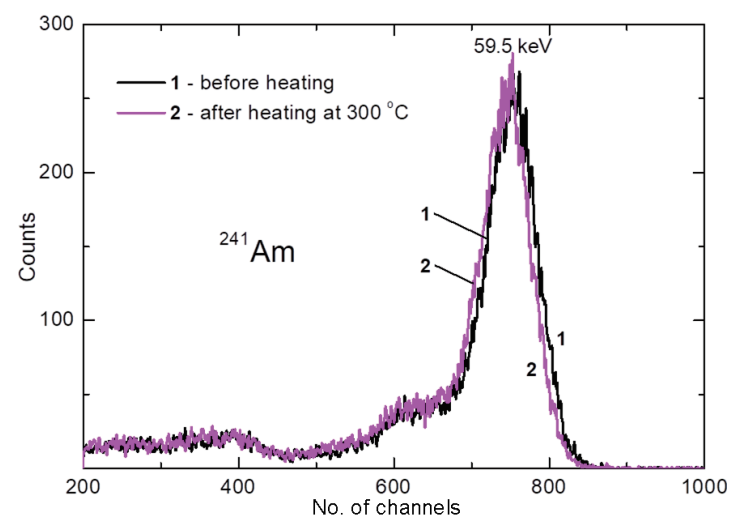

Fig. 5. (Color online) Spectra of ${ }^{241} \mathrm{Am}$ radioisotope obtained using $\mathrm{In} / \mathrm{CdTe} / \mathrm{Au}$ diode detector with $p-n$ junction before (curve 1) and after (curve 2) heat treatment at $300{ }^{\circ} \mathrm{C}$.

melting point [Figs. 4(b) and 5(b)] is attributed to the multilayered structure of the diode and the location of the electrical barrier in the deeper region (b) compared with the In/CdTe/Au Schottky diode (a) (Fig. 2). As illustrated in Fig. 2(b), the diode, formed by laser-induced backside doping, consists of the In electrode, In $/ n$-CdTe ohmic contact, low-resistivity In-doped $n$-CdTe layer (about $50 \mathrm{~nm}$ thick), built-in $p-n$ junction, high-resistivity $p$-like CdTe bulk, near-ohmic Au/p-CdTe contact, and Au electrode.

Thus, the deposited In film (electrode) is located on the low-resistivity $n$-type CdTe surface region doped with In atoms [Fig. 2(b)]. The In electrode and the surface of the $n$-CdTe:In layer form an ohmic contact. If the In film (electrode) is melted under the heating of the $\mathrm{In} / \mathrm{CdTe} / \mathrm{Au}$ $p-n$ junction diode at $T>T_{\text {In }}$ and then solidified, the electrical properties of the $\operatorname{In} / n$-CdTe:In interface are unchanged. The electrical contact between the metal and low-resistivity highly doped surface layer of the semiconductor remains ohmic [Fig. 2(b)].

It is a fact that the electrical potential barrier ( $p-n$ junction) located inside the CdTe semiconductor at a certain depth below the In/CdTe interface explains the absence of the thermal deterioration of the electrical and spectral properties of the $\mathrm{In} / \mathrm{CdTe} / \mathrm{Au}$ diodes, formed by laser-induced backside doping, and their tolerance to heating at temperatures lower and higher than the In melting point [Figs. 4(b) and 5(b)].

\section{Conclusions}

In this study, we examined the thermal stability of the electrical and spectral properties of $\mathrm{In} / \mathrm{CdTe} / \mathrm{Au} \mathrm{X}$ - and $\gamma$-ray detectors, namely, diodes with a Schottky barrier and those with a $p-n$ junction, which were fabricated using identical CdTe crystals and the same technological procedures except laser irradiation. Despite the fact that both types of diode had similar electrical and spectral characteristics, heating the samples, particularly at temperatures above the In melting point, led to rather different results. The characteristics of the In/CdTe/Au Schottky diode detectors deteriorated and exhibited an increase in reverse dark current (leakage 
current). Moreover, the $\mathrm{X}$ - and $\gamma$-ray sensitivities of such detectors completely disappeared after heat treatments, which resulted in the melting of the In electrode (rectifying Schottky contact). Certainly, the $\mathrm{Al}$ electrode used as a rectifying contact in CdTe-based Schottky diode detectors is not melted at such temperatures. However, the deposition of $\mathrm{Al}$ is more difficult than that of In; nonetheless, the heating of Schottky diodes degrades their characteristics. ${ }^{(21)}$

The In/CdTe/Au diode detectors with a $p-n$ junction, formed by laser-induced backside doping, were more resistant to heat treatment and demonstrated practically the same $I-V$ characteristics before and after heating at temperatures below and above the In melting point. The ideality factor of such diodes was stable with a value of approximately 2.8. The spectral characteristics of the untreated and heat-treated $p-n$ junction diode detectors, measured using an ${ }^{241} \mathrm{Am}$ radioisotope, exhibited almost the same detection efficiency and energy resolution.

The excellent thermal resistance of the $\mathrm{In} / \mathrm{CdTe} / \mathrm{Au} p-n$ junction diode detectors has enabled postprocessing and tiling, which require the heating of samples. This finding opens opportunities for the application of various bonding and bumping procedures for such $\mathrm{X}$ - and $\gamma$-ray detectors, which are expected to be useful in creating pixel matrices, forming tiled arrays, assembling large sensor modules, and developing other devices for imaging. In the future, we intend to reduce the pixel sizes of these detectors in order to use them as imaging sensors.

\section{Acknowledgments}

We would like to thank Dr. K. Takagi and Dr. A. Koike from ANSeeN Inc. for the useful discussions.

\section{References}

1 P. N. Cooper: Introduction to Nuclear Radiation Detectors (Cambridge University Press, Cambridge, 2011) p. 152.

2 R. Triboulet and P. Siffert: CdTe and Related Compounds; Physics, Defects, Hetero- and Nano-structures, Crystal Growth, Surfaces and Applications, Part II: Crystal Growth, Surfaces and Applications (Elsevier, Amsterdam, 2010) 1st ed., p. 417. https://doi.org/10.1016/C2009-0-61369-6

3 S. Del Sordo, L. Abbene, E. Caroli, A. M. Mancini, A. Zappettini, and P. Ubertini: Sensors 9 (2009) 3491. https://doi.org/10.3390/s90503491

4 L. A. Kosyachenko, T. Aoki, C. P. Lambropoulos, V. A. Gnatyuk, E. V. Grushko, V. M. Sklyarchuk, O. L. Maslyanchuk, O. F. Sklyarchuk, and A. Koike: IEEE Trans. Nucl. Sci. 60 (2013) 2845. https://doi.org/10.1109/ TNS.2013.2260356

5 C. P. Lambropoulos, T. Aoki, J. Crocco, E. Dieguez, C. Disch, A. Fauler, M. Fiederle, D. S. Hatzistratis, V. A. Gnatyuk, K. Karafasoulis, L. A. Kosyachenko, S. N. Levytskyi, D. Loukas, O. L. Maslyanchuk, A. Medvids, T. Orphanoudakis, I. Papadakis, A. Papadimitriou, C. Potiriadis, T. Schulman, V. M. Sklyarchuk, K. Spartiotis, G. Theodoratos, O. I. Vlasenko, K. Zachariadou, and M. Zervakis: IEEE Trans. Nucl. Sci. 58 (2011) 2363. https:// doi.org/10.1109/TNS.2011.2162964

6 T. Aoki, Yu. Ishida, D. Sakashita, V. A. Gnatyuk, A. Nakamura, Y. Tomita, Y. Hatanaka, and J. Temmyo: Proc. SPIE 5540, Hard X-Ray and Gamma-Ray Detector Physics VI (SPIE, 2004) 196-205. https://doi. org $/ 10.1117 / 12.559479$

7 V. A. Gnatyuk, T. Aoki, E. V. Grushko, L. A. Kosyachenko, and O. I. Vlasenko: Proc. SPIE 8142, Hard X-ray, Gamma-Ray, and Neutron Detector Physics XIII (SPIE, 2011) 81420B-1 7. https://doi.org/10.1117/12.895555

8 Hamamatsu Photonics: https://www.hamamatsu.com/jp/en/product/type/C9732DK-11/index.html

9 H. Shiraki, M. Funaki, Y. Ando, A. Tachibana, S. Kominami, and R. Ohno: IEEE Trans. Nucl. Sci. 56 (2009) 1717. https://doi.org/10.1109/TNS.2009.2016843 
10 S. Denda: High-Dimensional Semiconductor Technology - 3D/2.5D/2.1D Mounting Through Electrodes (Tokyo Denki University Press, Tokyo, 2015) p. 144.

11 G. Harman: Wire Bonding in Microelectronics (McGraw-Hill, New York, 2010) 3rd ed., p. 427.

12 T. Takahashi, S. Watanabe, G. Sato, Y. Okada, S. Kubo, Y. Kuroda, M. Onishi, and R. Ohno: IEEE Trans. Nucl. Sci. 48 (2001) 287. https://doi.org/10.1109/23.940067

13 R. Luhta, M. Chappo, B. Harwood, R. Mattson, D. Salk, and C. Vrettos: Proc. SPIE 6142, Medical Imaging 2006: Physics of Medical Imaging (SPIE, 2006) 61420U-1 12. https://doi.org/10.1117/12.653245

14 M. Motoyoshi and M. Koyanagi: J. Instrum. 4 (2009) P03009. https://doi.org/10.1088/1748-0221/4/03/P03009

15 S. M. Sze and K. K. Ng: Physics of Semiconductor Devices (Wiley, New Jersey, 2007) 3rd ed., Chaps. 2-3, pp. 79-196. https://doi.org/10.1002/0470068329

16 K. S. Zelenska, D. V. Gnatyuk, and T. Aoki: J. Laser Micro/Nanoeng. 10 (2015) 298. https://doi.org/10.2961/ jlmn.2015.03.0011

17 K. S. Zelenska, D. V. Gnatyuk, and T. Aoki: Proc. 2015 IEEE Nuclear Science Symposium and Medical Imaging Conference (IEEE, 2015) 1-2. https://doi.org/10.1109/NSSMIC.2015.7582277

18 K. Zelenska, D. Gnatyuk, and T. Aoki: Proc. 2016 IEEE Nuclear Science Symposium, Medical Imaging Conference and Room-Temperature Semiconductor Detector Workshop (IEEE, 2016) 1-3. https://doi. org/10.1109/NSSMIC.2016.8069953

19 J. Nishizawa, H. Nakagawa, K. Zelenska, V. Gnatyuk, A. Koike, and T. Aoki: Proc. 2019 IEEE Nuclear Science Symposium and Medical Imaging Conference (IEEE, 2019) 1-4. https://doi.org/10.1109/NSS/ MIC42101.2019.9059751

20 V. M. Sklyarchuk, V. A. Gnatyuk, and T. Aoki: IEEE Trans. Nucl. Sci. 66 (2019) 2140. https://doi.org/10.1109/ TNS.2019.2935836

21 F. Principato, A. A. Turturici, M. Gallo, and L. Abbene: Nucl. Instrum. Methods Phys. Res., Sect. A 730 (2013) 141. https://doi.org/10.1016/j.nima.2013.05.157

22 S. E. Zelensky, L. V. Poperenko, A. V. Kopyshinsky, and K. S. Zelenska: Proc. SPIE 8434, Nonlinear Optics and Applications VI (SPIE, 2012) 84341H-1 6. https://doi.org/10.1117/12.921999

23 L. A. Kosyachenko, V. M. Sklyarchuk, O. F. Sklyarchuk, and V. A. Gnatyuk: Semicond. Sci. Technol. 22 (2007) 911. https://doi.org/10.1088/0268-1242/22/8/015

24 K. Harada, A. G. Werner, M. Pfeiffer, C. J. Bloom, C. M. Elliott, and K. Leo: Phys. Rev. Lett. 94 (2005) 036601. https://doi.org/10.1103/PhysRevLett.94.036601

25 F. Meillaud, A. Shah, C. Droz, E. Vallat-Sauvain, and C. Miazza: Sol. Energy Mater. Sol. Cells 90 (2006) 2952. https://doi.org/10.1016/j.solmat.2006.06.002

\section{About the Authors}

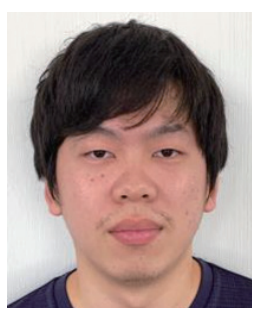

Junichi Nishizawa received his B.S. and M.S. degrees from Shizuoka University, Japan, in 2015 and 2017, respectively. Since 2017, he has been a postgraduate student at the Graduate School of Science and Technology, Shizuoka University. His research interests are in CdTe-based and scintillator high-energy detectors, laser-induced doping, the formation and characterization of Schottky and $p-n$ junction diodes, $\mathrm{X} / \gamma$-ray pixel sensors, and imaging devices. (nishizawa.junichi.15@shizuoka.ac.jp)

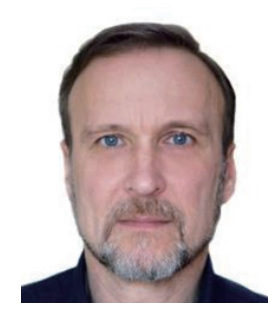

Volodymyr Gnatyuk graduated from the Faculty of Physics, V. N. Karazin Kharkiv National University in 1985 and received his Ph.D. degree from the Institute of Semiconductor Physics of the NAS of Ukraine (ISP-NASU) in 1993. He became an associate professor of Poltava National Pedagogical University in 1995 (Ukraine) and an honorable guest professor of Shizuoka University, Japan, in 2009-2020. Since 2004, he has been a senior scientist at ISP-NASU. His research interests are in semiconductors, optics, photooelectronics, and sensors. (gnatyuk@ua.fm) 


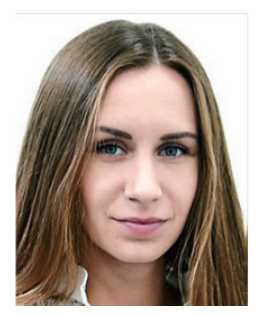

Kateryna Zelenska received her B.S. and M.S. degrees in physics, and her B.S. and Ph.D degrees in biology, from Taras Shevchenko National University of Kyiv (TSNUK), Ukraine, in 2009, 2011, 2012, and 2016, respectively. She was an engineer at TSNUK (2016-2018) and a postdoctoral researcher at Shizuoka University, Japan (2018-2020). Since 2020, she has been an academic researcher at Shizuoka University, Japan. Her research interests are in material science, semiconductor devices, optics, and quantum electronics. (czelenska@gmail.com)

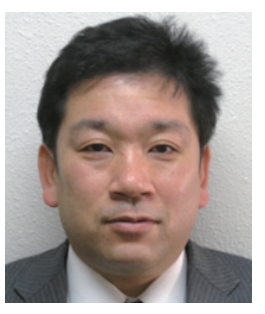

Toru Aoki received his B.Eng., M.Eng., and Ph.D. degrees from Shizuoka University, Japan, in 1991, 1993, and 1996, respectively. From 1996, he was an assistant professor and an associate professor at the Research Institute of Electronics, Shizuoka University. Since 2015, he has been a professor at Shizuoka University. He is also CTO at ANSeeN Inc. (since 2011). He is a special advisor to the President of Shizuoka University. His research interests are in material engineering, semiconductor devices, $\mathrm{X} / \gamma$-ray detectors, and CT. (aoki.toru@shizuoka.ac.jp) 